\title{
On Performance Evaluation of Advance Irregular Alpha Multi-Stage Interconnection Network-2
}

\author{
Abhimanyu Bhardwaj \\ Department of \\ Information Technology \\ Suresh Gyan Vihar University \\ Jaipur, India
}

\author{
Savita Shiwani \\ Department of \\ Information Technology \\ Suresh Gyan Vihar University \\ Jaipur, India
}

\begin{abstract}
In this paper, the author has proposed a new multi-stage interconnection network named as advance irregular alpha multi-stage interconnection network-2 (AIAMIN-2). The AIAMIN-2 is a single switch fault tolerant network. It is compared with modified alpha network (MALN) and irregular modified augmented baseline network (IMABN). Results show that AIAMIN-2 performs better than the MALN and IMABN in faulty and non-faulty conditions.
\end{abstract}

\section{Keywords}

Interconnection Network; Multi-Stage Interconnection Network; MALN; IMABN, AIAMIN-2; Fault

\section{INTRODUCTION}

Multi-stage interconnection (MIN) provides the reliable and fast communication to the parallel processing applications. Faults in MIN prevent the data transmission process [1-14]. Therefore, designing a good fault tolerant MIN is the key concern. In this paper the author has proposed the AIAMIN-2 which is single switch fault tolerant. Single switch fault tolerant means network can sustain a faulty switch in stage simultaneously. Further, performance of AIAMIN-2 is compared with MALN [13] and IMABN [14] in faulty and non-faulty network conditions. Here, "faulty" means single switch is faulty in every stage of network [15-30].

\section{PROPOSED INTERCONNECTION NETWORK}

The structure of AIAMIN-2 is shown in Figure 1. This network has 16 sources and 16 destinations. In Figure 1, the source, destination, multiplexers and demultiplexers are represented by S, D, Mux and Demux respectively. There are 16 Mux and 16 Demux in AIAMIN-2. The size of each Mux and Demux is $2 \times 1$ and $1 \times 2$ respectively. Each stage consists of 8 switching elements (SEs) except the second stage. The size of each SE in first and last stage is $2 \times 3$ and $5 \times 2$ respectively. The second stage consists of 4 SEs and size of each SE is $4 \times 8$.

In Figure 1, it can be observed that the SEs of first stage are connected with sources through Mux and the SEs of second stage. The SEs of second stage connects the first and third stage. Further, the SEs of third stage are connected with the destinations through Demux. In AIAMIN-2, SEs are categorized in 3 category i.e. main SE, first alternate SE and second alternate SE as each source is connected with 3 SEs via Mux e.g. source 0 is connected with $\mathrm{SE}$ a, e and f. It shows a, e and $f$ are the main SE, first alternate SE and second alternate $\mathrm{SE}$ respectively.

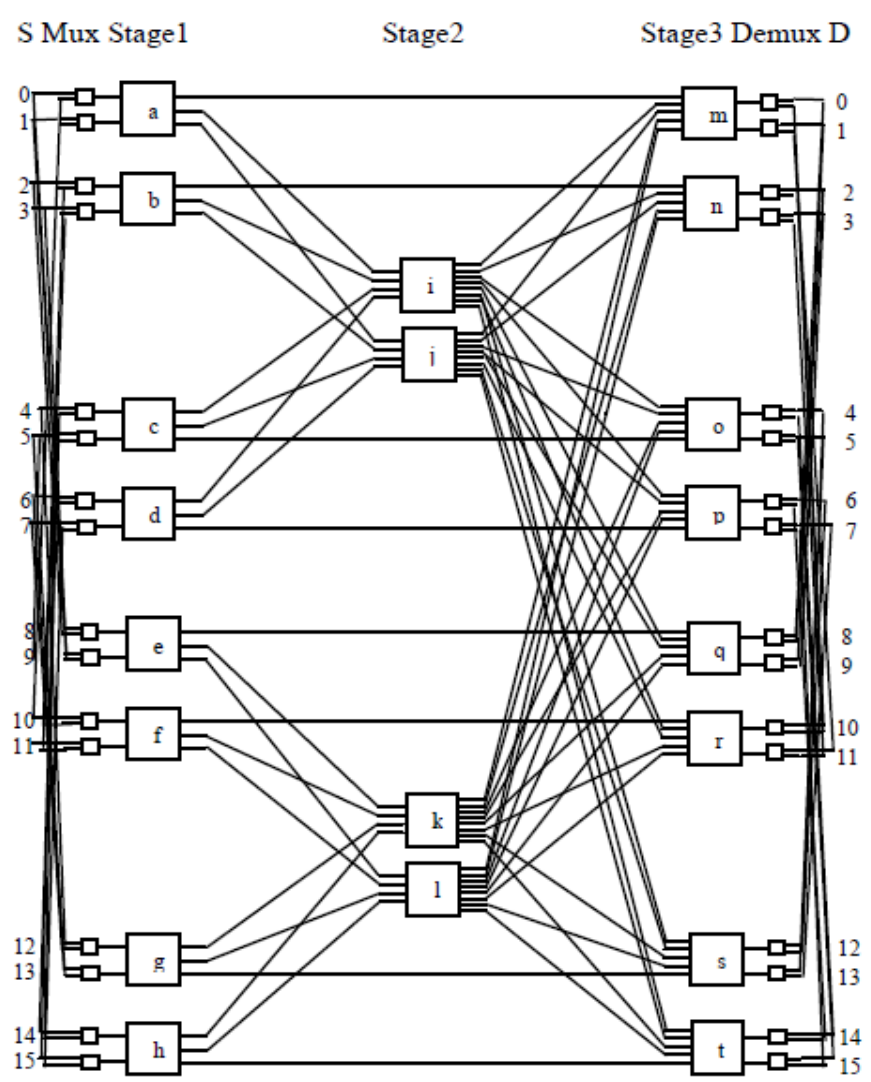

Fig. 1: 16*16 AIAMIN-2

Similarly, the main SE, first alternate SE and second alternate SE for other sources can be obtained. In second stage, SEs i and $\mathrm{j}$ are primary and secondary SEs respectively for the SEs a, b, c and d. Similarly, SEs k and 1 are primary and secondary SEs for SEs e, $f, g$ and $h$. Further, in third stage we have main SE, first alternate SE and second alternate SE e.g. for destination 0 , SEs $\mathrm{m}, \mathrm{q}$ and $\mathrm{r}$ are the main SE, first alternate $\mathrm{SE}$ and second alternate SE respectively. Similarly, the main $\mathrm{SE}$, first alternate SE and second alternate SE for other destinations can be obtained.

Table 1 shows the various symbols and their meaning which are used throughout the paper. 
Table 1. Symbol table

\begin{tabular}{|c|c|}
\hline Symbol & Meaning of Symbol \\
\hline $\mathrm{MSE}_{1}$ & Main SE of first stage \\
\hline $\mathrm{FAS}_{1}$ & First alternate SE of first stage \\
\hline $\mathrm{SAS}_{1}$ & Second alternate SE of first stage \\
\hline $\mathrm{PSE}_{2}$ & Primary SE of second stage \\
\hline $\mathrm{SSE}_{2}$ & Secondary SE of second stage \\
\hline $\mathrm{MSE}_{3}$ & Main SE of third stage \\
\hline $\mathrm{FAS}_{3}$ & First alternate SE of third stage \\
\hline $\mathrm{SAS}_{3}$ & Second alternate SE of third stage. \\
\hline
\end{tabular}

\subsection{Routing Algorithm of AIAMIN-2}

Algorithm_AIAMIN-2 is the routing algorithm of AIAMIN-2. According to this algorithm, initially data packets arrive on the main SE of first stage. If it is faulty then data packets will be received by the first alternate SE of first stage. If it is also faulty then data packets will be collected by the second alternate SE of first stage. If all the required SEs are faulty then network will fail otherwise anyone of the SE i.e. $\mathrm{MSE}_{1}$ or $\mathrm{FAS}_{1}$ or $\mathrm{SAS}_{1}$ will collect the data packets.

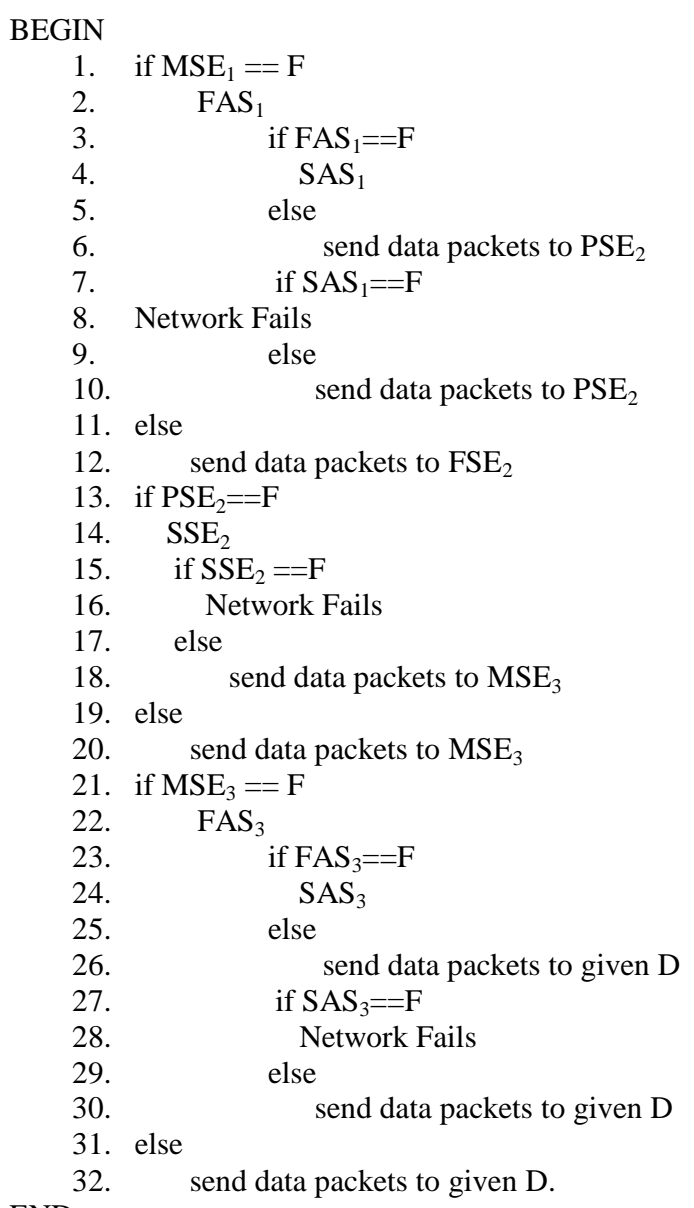

Further, data packets will be sent to primary SE of second stage. If it is faulty then data packets will be sent to secondary SE of second stage. If both of the SEs are faulty then communication is not possible and network will fail otherwise data packets will be sent to appropriate SE of third stage. In third stage, data packets will be collected by the non-faulty SE. If all the required SE are faulty then network will fail otherwise data packets will be transmitted to the given destination through Demux.

\section{PERFORMANCE EVALUATION PARAMETERS}

These are the basic factors which are used in order to measure the performance of any network:

\subsection{Bandwidth (BW)}

It can be defined as follows:

" $B W$ is defined as the mean number of active memory modules in a transfer cycle of interconnection networks (INs) and therefore, $B W$ is the total number of request matured [11, 13]".

If there are $a^{\mathrm{n}}$ sources and $\mathrm{b}^{\mathrm{n}}$ destinations then bandwidth will be as follows:

$\mathrm{BW}=\mathrm{b}^{\mathrm{n}} \times \mathrm{p}_{\mathrm{n}}$

Here $p_{n}$ is the request generation probability or load factor. Probability equations for MALN are:

$\mathrm{p}_{1}=1-\left(1-\mathrm{p}_{0} / 3\right)^{\wedge} 3$

$\mathrm{p}_{2}=1-\left(1-\mathrm{p}_{1} / 6\right)^{\wedge} 3$

$\mathrm{p}_{3}=1-\left(1-\mathrm{p}_{2} / 3\right)^{\wedge} 3$

$\mathrm{p}_{4}=1-\left\{\left(1-\mathrm{p}_{3}\right) \times\left(1-\mathrm{p}_{1} / 2\right)\right\}^{\wedge} 2$

BW_MALN=N $\times \mathrm{p}_{4}$

Probability equations for IMABN are:

$\mathrm{p}_{1}=1-\left(1-\mathrm{p}_{0} / 3\right)^{\wedge} 3$

$\mathrm{p}_{2}=1-\left(1-\mathrm{p}_{1} / 10\right)^{\wedge} 5$

$\mathrm{p}_{3}=1-\left\{\left(1-\mathrm{p}_{2}\right) \times\left(1-\mathrm{p}_{1} / 2\right)\right\}^{\wedge} 2$

$\mathrm{BW} \_\mathrm{IMABN}=\mathrm{N} \times \mathrm{p}_{3}$

Probability equations for AIAMIN-2 are:

$\mathrm{p}_{1}=1-\left(1-\mathrm{p}_{0} / 3\right)^{\wedge} 2$

$\mathrm{p}_{2}=1-\left(1-\mathrm{p}_{1} / 8\right)^{\wedge} 4$

$\mathrm{p}_{3}=1-\left\{\left(1-\mathrm{p}_{2}\right) \times\left(1-\mathrm{p}_{1} / 2\right)\right\}^{\wedge} 5$

BW_AIAMIN-2=N $\times \mathrm{p}_{3}$

\subsection{Probability of Acceptance (PA)}

It can be defined as follows:

"PA is defined as ratio of bandwidth to the expected number of requests generated per transfer cycle [13, 14]". Formula for PA is:

$\mathrm{PA}=(\mathrm{BW} / \mathrm{N} \times \mathrm{p})$

\subsection{Throughput (TP)}

It can be defined as follows: 
"TP means average number of cells delivered by a network per unit time [11-14]".

Formula for TP is:

\section{$\mathrm{TP}=(\mathrm{BW} / \mathrm{N} \times \mathrm{t})$}

Here $t$ is the transmission time of data packets. Transmission time is the time which is taken by the data packets from the given source to the given destination.

\subsection{Processor Utilization (PU)}

It can be defined as follows:

"PU is defined as percentage of time the processor is active doing computation without accessing the global memory [1114]".

Formula for PU is:

$\mathrm{PU}=(\mathrm{BW} / \mathrm{N} \times \mathrm{p} \times \mathrm{t})$

\subsection{Processing Power (PP)}

It can be defined as follows:

"PP is defined as sum of processor utilization over the number of processors [11-14]".

Formula for PP is:

$\mathrm{PP}=(\mathrm{N} \times \mathrm{PU})$

\section{RESULTS AND DISCUSSIONS}

On the basis of above discussed formulas the author has measured the performance of MALN [13], IMABN [14] and AIAMIN-2. The transmission time is also the important factor in this simulation. The author has assumed that a data packet takes 1 second from one node to another node. Here node may be any source or destination or SE. The other thing is that, if a node is faulty then data packet takes one second extra to reach from one node to other node. Based on this assumption, the author has obtained the following results:

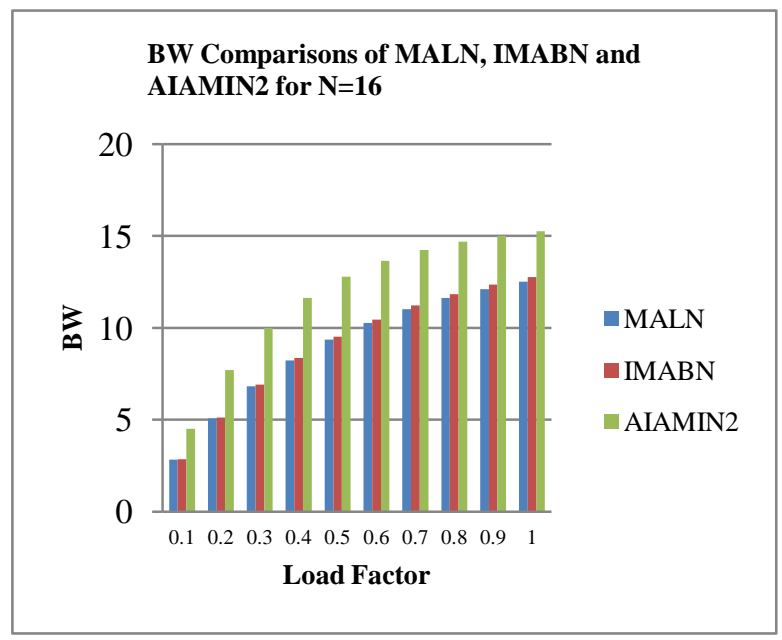

Fig.2: BW Comparisons

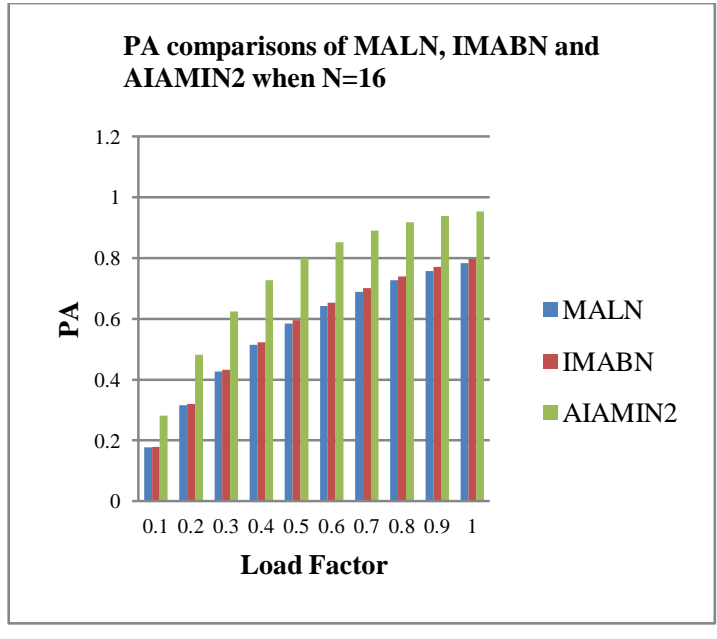

Fig.3: PA Comparisons

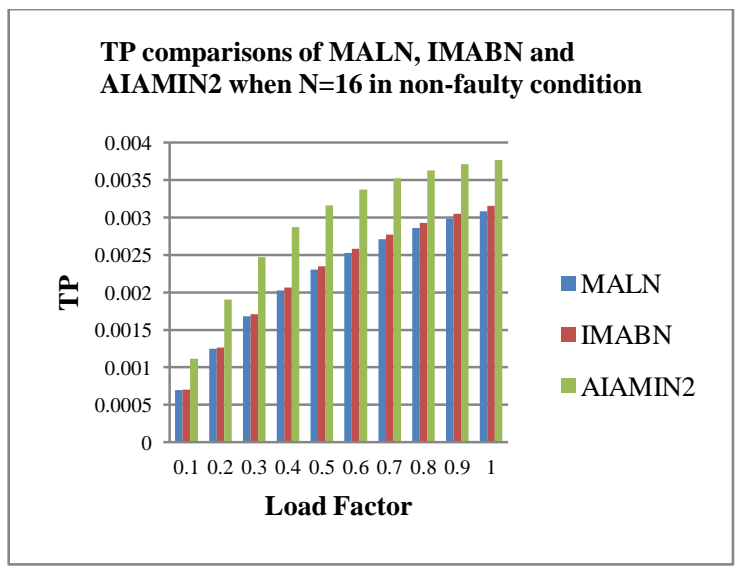

Fig.4: TP Comparisons in Non-Faulty Condition

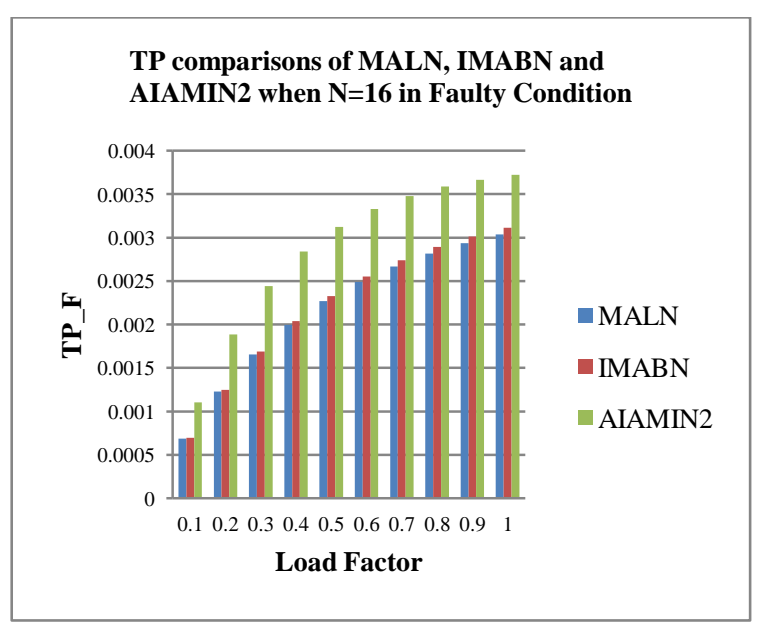

Fig.5: TP Comparisons in Faulty Condition 


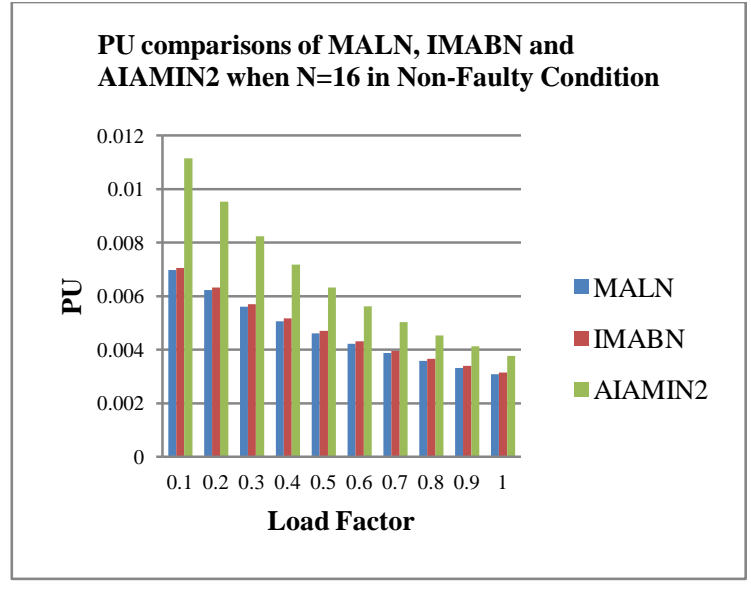

Fig.6: PU Comparisons in Non-Faulty Condition

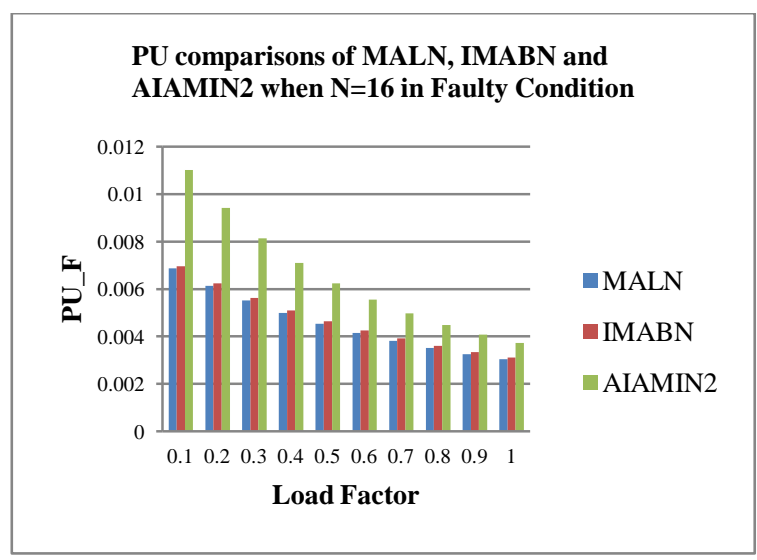

Fig.7: PU Comparisons in Faulty Condition

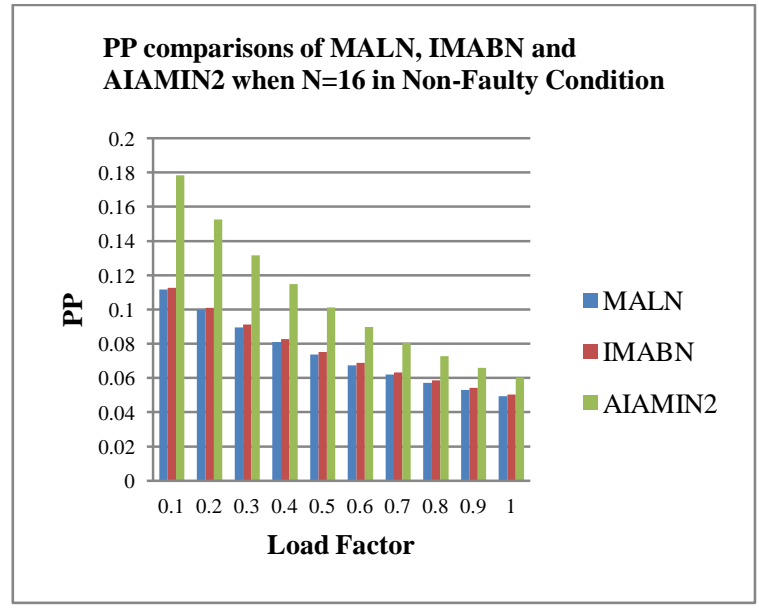

Fig.8: PP Comparisons in Non-Faulty Condition

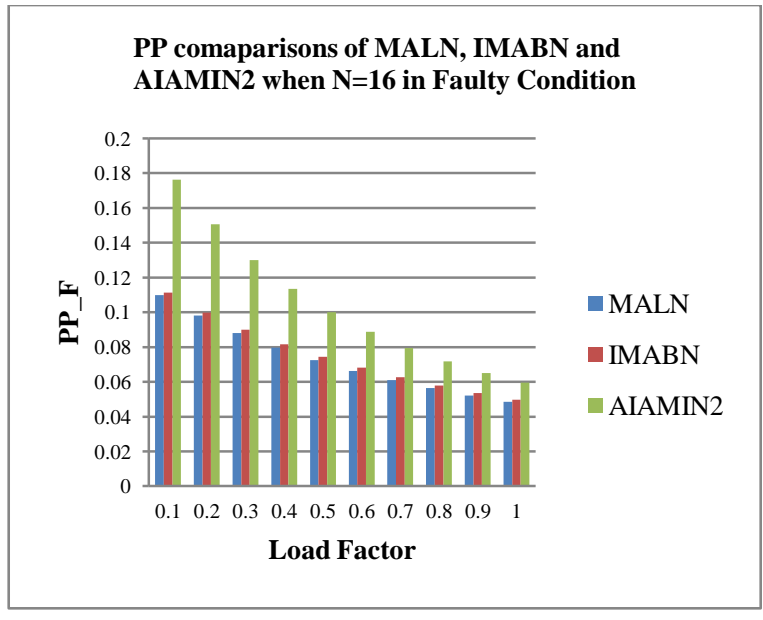

Fig.9: PP Comparisons in Faulty Condition

In all the figures i.e. from figure 2 to 9 , it is observed that the AIAMIN-2 gives better results than the MALN and IMABN in faulty and non-faulty network situations.

\section{CONCLUSION AND FUTURE SCOPE OF WORK}

In this research work, the author has shown the comparison of performance of MALN, IMABN and AIAMIN-2. On all the factors of performance, AIAMIN-2 performs better than the MALN and IMABN in faulty and non-faulty condition. Further, AIAMIN-2 is a good fault tolerant network; it can sustain single faulty SE in each simultaneously and does the data transmission process perfectly. In future, the proposed network model can be developed and analyzed in a generalized way.

\section{REFERENCES}

[1] K. Hwang, Advanced Computer Architecture: Parallelism, Scalability, Programmability, Tata McGrawHill, India, ISBN 0-07-053070-X, 2000.

[2] J. Duato, S. Yalamanchili and L.M. Ni, Interconnection Networks: An Engineering Approach, Morgan Kaufmann, ISBN 1-55860-852-4, 2003.

[3] W. Dally and B. Towles, Principles and Practices of Interconnection Networks, Morgan Kaufmann, San Francisco, ISBN 978-0-12-200751-4, 2004.

[4] Chenggong Charles Fan and Jehoshua Bruck, Tolerating Multiple Faults in Multistage Interconnection Networks with Minimal Extra Stages, IEEE Transactions on Computers, Volume 49, Number 9, pp. 998-1004, 2000.

[5] John Garofalakis and Eleftherios Stergiou, An Approximate Analytical Performance Model for Multistage Interconnection Networks with Backpressure Blocking Mechanism, Journal of Communications, Volume 5, Number 3, pp. 247-261, 2010.

[6] Ved Prakash Bhardwaj, Nitin and Vipin Tyagi, An Algorithmic Approach to Minimize the Conflicts in an Optical Multistage Interconnection Network, Communications in Computer and Information Science Series (CCIS), Springer-Verlag, Volume 191, ISSN: 1865:0929, pp. 568-576, 2011.

[7] Ved Prakash Bhardwaj and Nitin, Applying Address Selection Algorithm on Nonblocking Optical Multi-stage Interconnection Network, Proceedings of the IEEE 
World Congress on Information and Communication Technologies, Mumbai, INDIA, pp. 694-698, 2011.

[8] Dimitris C. Vasiliadis, George E. Rizos and Costas Vassilakis, Performance Analysis of Dual-Priority Multilayer Multistage Interconnection Networks under Multicast Environment, Journal of Networks, Volume 6, Number 6, pp. 858-871, 2011.

[9] Minsu Choi, Nohpill Park, and Fabrizio Lombardi, Modeling and Analysis of Fault Tolerant Multistage Interconnection Networks, IEEE Transactions on Instrumentation and Measurement, Volume 52, Number 5, pp. 1509-1519, 2003.

[10] D. C. Vasiliadis, G. E. Rizos and C. Vassilakis, ClassBased Weighted Fair Queuing Scheduling on DualPriority Delta Networks, Journal of Computer Networks and Communications, DOI:10.1155/2012/859694, Volume (2012) Hindawi Publishing Corporation, 2012.

[11] Jyotsna Sengupta, Interconnection Networks for Parallel Processing, IS

BN 81-7629-736-4, 2005.

[12] Rita Mahajan and Renu Vig, Performance and Reliability Analysis of New Fault Tolerant Advance Omega Network, WSEAS Transactions on Computers, Volume 7, pp. 1280-1290, 2008.

[13] Amardeep Gupta and P K Bansal, Fault Tolerant Irregular Modified Alpha Network and Evaluation of Performance Parameters, International Journal of Computer Applications, Volume 4, Number 1, pp. 9-13, 2010.

[14] Mamta Ghai, Vinay Chopra and Karamjit Kaur Cheema, Performance Analysis of Fault-Tolerant Irregular Baseline Multistage Interconnection Network, International Journal on Computer Science and Engineering, Volume 2, Number 9, pp. 3079-3084, 2010.

[15] Ved Prakash Bhardwaj and Nitin, A New Fault Tolerant Routing Algorithm for IASEN-2, Proceedings of the 2nd IEEE International Conference on Advances in Computing and Communications (IEEE ICACC), Kerala, INDIA, pp. 199-202, 2012.

[16] Nitin, On Asymptotic Analysis of Packet and Wormhole Switched Routing Algorithm for Application-Specific Networks-On-Chip, Journal of Computer and Electrical Engineering, Hindawi Publishing Corporation, Volume 2012, DOI:10.1155/2012/216406, pp. 1-27, 2012.

[17] Shaily Mittal and Nitin, Memory Map: A Multiprocessor Cache Simulator, Journal of Electrical and Computer Engineering, Hindawi Publishing Corporation, Volume 2012, DOI:10.1155/2012/365091, pp. 1-12, 2012.

[18] Nitin and Durg Singh Chauhan, Stochastic Communication for Application Specific Networks-onChip, Journal of Supercomputing, Springer, Volume 59, Number 2, DOI: $10.1007 / \mathrm{s} 11227-010-0472-5$, pp. 779810,2010 .

[19] Nitin and Durg Singh Chauhan, Comparative Analysis of Traffic Patterns on k-ary n-tree using Adaptive Algorithms based on Burton Normal Form, Journal of
Supercomputing, Springer, Volume 59, Number 2, DOI: 10.1007/s11227-010-0454-7, pp. 569-588, 2010

[20] Nitin, Shruti Garhwal and Neha Srivastava, Designing a Fault-tolerant Fully-chained Combining Switches Multistage Interconnection Network with Disjoint Paths, The Journal of Supercomputing, Springer, Volume 55, Number 3, DOI 10.1007/s11227-009-0336-z, pp. 400431, 2009.

[21] Nitin and Ashok Subramanian, Efficient Algorithms to Solve Dynamic MINs Stability Problems using Stable Matching with Complete TIES, Journal of Discrete Algorithms, Elsevier, Volume 6, Issue 3, pp. 353-380, 2008.

[22] Nitin, Component Level Reliability Analysis of Faulttolerant Hybrid MINs, WSEAS Transactions on Computers, ISSN: 1109-2750, Issue 9, Volume 5, pp. 1851-1859, 2006.

[23] Nitin and Durg Singh Chauhan, A New Fault Tolerant Routing Algorithm for MALN-2, Communications in Computer and Information Science, Springer-Verlag, pp. 247-254, 2012.

[24] Ching-Wen Chen and Chung-Ping Chung, Designing A Disjoint Paths Interconnection Network with Fault Tolerance and Collision Solving, The Journal of Supercomputing, 34, pp. 63-80, 2005.

[25] Ved Prakash Bhardwaj and Nitin, A New Fault Tolerant Routing Algorithm for Advance Irregular Alpha Multistage Interconnection Network, Advances in Intelligent and Soft Computing, Springer-Verlag, ISSN: 1867-5662, pp. 979-987, 2012.

[26] Ved Prakash Bhardwaj and Nitin, A New Fault Tolerant Routing Algorithm for Advance Irregular Alpha Multistage Interconnection Network, Advances in Intelligent and Soft Computing, Springer-Verlag, ISSN: 1867-5662, pp. 979-987, 2012.

[27] B.V.Suresh Kumar, M.Venkata Rao and M.A.Ram Prasad, Adaptive Fault Tolerant Routing In Interconnection Networks: A Review, Int. J. Advanced Networking and Applications, Volume: 02, Issue: 06, pp. 933-940, 2011.

[28] Srinivasan Murali, David Atienza, Luca Benini and Giovanni DeMicheli, A Method for Routing Packets Across Multiple Paths in NoCs with In-Order Delivery and Fault-Tolerance Gaurantees, VLSI Design, doi:10.1155/2007/37627,Volume (2007) Hindaw Publishing Corporation, 2007.

[29] Nitin and Durg Singh Chauhan, A New Fault-Tolerant Routing Algorithm for IMABN-2, Proceedings of the 2nd IEEE International Conference on Advances in Computing and Communications (IEEE ICACC), Kerala, INDIA, pp. 215-218, 2012.

[30] Ved Prakash Bhardwaj and Nitin, A New Fault Tolerant Routing Algorithm for Advance Irregular Augmented Shuffle Exchange Network, Proceedings of the 14th IEEE International conference on Computer Modeling and Simulation (IEEE UKSIM), Emmanuel College, Cambridge, UK, pp. 505-509, 2012. 